\title{
Tomographic RF Spectroscopy of a Trapped Fermi Gas at Unitarity
}

\author{
Y. Shin* C. H. Schunck, A. Schirotzek, and W. Ketterle \\ Department of Physics, MIT-Harvard Center for Ultracold Atoms, and Research Laboratory of Electronics, \\ Massachusetts Institute of Technology, Cambridge, Massachusetts, 02139
}

(Dated: November 5, 2018)

\begin{abstract}
We present spatially resolved radio-frequency spectroscopy of a trapped Fermi gas with resonant interactions and observe a spectral gap at low temperatures. The spatial distribution of the spectral response of the trapped gas is obtained using in situ phase-contrast imaging and 3D image reconstruction. At the lowest temperature, the homogeneous $\mathrm{rf}$ spectrum shows an asymmetric excitation line shape with a peak at $0.48(4) \varepsilon_{F}$ with respect to the free atomic line, where $\varepsilon_{F}$ is the local Fermi energy.
\end{abstract}

PACS numbers: 03.75.Ss, 03.75.Hh, 32.30.Bv

Ultracold Fermi gases near a Feshbach resonance provide new insight into fermionic superfluidity, allowing the study of the crossover from Bardeen-Cooper-Schrieffer (BCS) superfluids of Cooper pairs to Bose-Einstein condensates (BECs) of dimer molecules. Many aspects of the BEC-BCS crossover, including superfluidity [1], have been experimentally investigated [2]. The properties of Fermi gases on resonance at unitarity, where the amplitude of the scattering length between the fermion atoms diverges to infinity and the system shows universal behavior [3], are of great importance to understand the crossover physics. Precision measurements of the critical temperature [4], the interaction energy [5] and collective excitations [6] have presented stringent quantitative test to the theoretical description of strongly interacting Fermi gases.

Radio-frequency (rf) spectroscopy measures an excitation spectrum by inducing transitions to different hyperfine spin states. This method has been employed in strongly interacting Fermi gases, leading to the observation of unitarity limited interactions [7, 8], molecule formation on the BEC side of the Feshbach resonance [9] as well as pairing in the crossover regime [10, 11]. Rf spectroscopy provides valuable information on the nature of the pairs. Since an rf photon can dissociate bound molecules or fermion pairs into the free atom continuum, the binding energy of the pairs or the excitation gap is determined. Furthermore the excitation line shape is related to the wave function of the pairs, e.g. larger pairs have narrower lines. However, currently all experimental measurements on the excitation spectrum in strongly interacting Fermi gases [10, 12] have been performed with samples confined in a harmonic trapping potential so that the spectral line shape is broadened due to the inhomogeneous density distribution of the trapped samples, preventing a more stringent comparison with theoretical predictions [13, 14, 15, 16].

In this Letter, we demonstrate spatially resolved $\mathrm{rf}$ spectroscopy of a trapped, population-balanced Fermi gas at unitarity at very low temperature. The spatial distribution of the rf-induced excited region in the trapped gas was recorded with in situ phase-contrast imaging [17] and the local rf spectra were compiled after 3D image reconstruction. In contrast to the inhomogeneous rf spectrum, the homogeneous local rf spectrum shows a clear spectral gap with an asymmetric line shape. We observe that the spectral peak shifts by $0.48(4) \varepsilon_{F}$ to higher energy and that the spectral gap is $0.30(8) \varepsilon_{F}$ with respect to the free atomic reference line, where $\varepsilon_{F}$ is the local Fermi energy. This new spectroscopic method overcomes the line broadening problem for inhomogeneous samples and provides homogeneous rf spectra of a resonantly interacting Fermi gas revealing the microscopic physics of fermion pairs.

We prepared a degenerate Fermi gas of spin-polarized ${ }^{6} \mathrm{Li}$ atoms in an optical trap, using laser cooling and sympathetic cooling with ${ }^{23} \mathrm{Na}$ atoms, as described in Ref. [18]. An equal mixture of the two lowest hyperfine states $|1\rangle$ and $|2\rangle$ (corresponding to the $\mid F=1 / 2, m_{F}=$ $1 / 2\rangle$ and $\left|F=1 / 2, m_{F}=-1 / 2\right\rangle$ states at low magnetic field) was created at a magnetic field $B=885 \mathrm{G}$. A broad Feshbach resonance located at $B_{0}=834 \mathrm{G}$ strongly enhanced the interactions between the two states. The final evaporative cooling by lowering the trap depth and all spectroscopic measurements were performed at $B=$ $833 \mathrm{G}$. The total atom number was $N_{t}=1.0 \pm 0.1 \times 10^{7}$ and the radial (axial) trap frequency was $f_{r}=129 \mathrm{~Hz}$ $\left(f_{z}=23 \mathrm{~Hz}\right)$. The Fermi energy (temperature) of a noninteracting equal mixture with the same total atom number is $E_{F}=h\left(f_{r}^{2} f_{z}\right)^{1 / 3}\left(3 N_{t}\right)^{1 / 3}=h \times 22.3 \mathrm{kHz}$ $\left(T_{F}=E_{F} / k_{B}=1.07 \mu \mathrm{K}\right)$, where $h$ is Planck's constant and $k_{B}$ is Boltzmann's constant. The ratio of the sample temperature $T$ to $T_{F}$ of $\approx 0.06$ was determined by fitting a finite temperature Thomas-Fermi (TF) distribution to the whole cloud after expansion.

Rf spectroscopy was performed by driving atoms in state $|2\rangle$ to the next lowest hyperfine state $|3\rangle$ (corresponding to $\left|F=3 / 2, m_{F}=-3 / 2\right\rangle$ at low field) that was initially empty. After applying an rf pulse of $1 \mathrm{~ms}$, we directly measured the in situ distribution of the density difference $n_{d} \equiv n_{1}-n_{2}$ in the excited sample, using a phase-contrast imaging technique [17], where $n_{1}$ and 
(a) $20 \mathrm{kHz}$

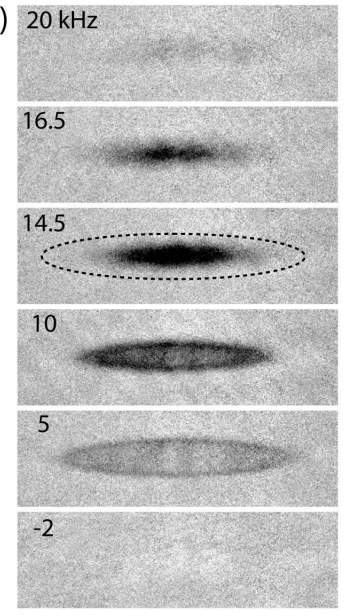

(b)

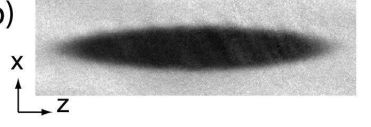

(c)

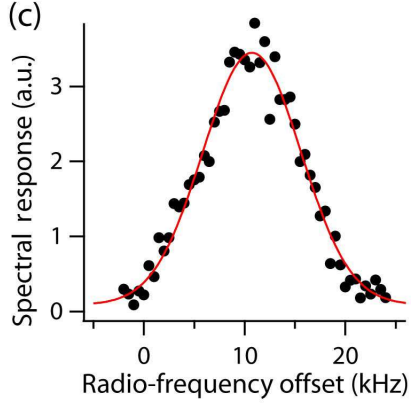

FIG. 1: (color online) Radio-frequency (rf) spectroscopy of a Fermi gas with in situ phase-contrast imaging. (a) After applying an rf pulse, the spatial distribution of the density difference between state $|1\rangle$ and $|2\rangle$ is recorded with the phasecontrast imaging technique [17]. The density depletion reflects the spin excitation induced by the rf pulse. The dashed line indicates the size of the trapped sample. (b) An absorption image of an equal mixture without applying an rf pulse. The field of view for each image is $205 \mu \mathrm{m} \times 680 \mu \mathrm{m}$. (c) Overall rf spectrum of the inhomogeneous sample is obtained by integrating the signal in the phase-contrast images. The red line is a Gaussian fit to the spectrum.

$n_{2}$ are the densities of atoms in the states $|1\rangle$ and $|2\rangle$, respectively. The frequency of the imaging beam was set to make the net phase shift in the sample proportional to the density difference $n_{d}$, which was done by zeroing the optical signal in an equal mixture without applying an rf pulse [17]. Since the initial atom densities in state $|1\rangle$ and $|2\rangle$ are equal, the density difference $n_{d}$ represents the atom number depletion in state $|2\rangle$, the spectral response $I$ [19].

The total spectral response, obtained by integrating over the phase contrast images, reproduces earlier results [10, 11]. The phase-contrast images now reveal the nature of the observed line shape (Fig. 11). The spectral response strongly depends on position. The inner region of the cloud, which is at higher density, shows a higher resonance frequency. The integrated inhomogeneous spectrum peaks at the rf offset $\Delta \nu_{i} \approx 10 \mathrm{kHz}[20]$. The spatially resolved images reveal that at this frequency, no excitations occur in the center of the cloud, but rather in a spatial shell. The rf offset $\Delta \nu$ is measured with respect to the resonance frequency of the $|2\rangle-|3\rangle$ transition in the absence of atoms in state $|1\rangle$ [21].

Local rf spectra $I(r, \Delta \nu)$ are compiled from the reconstructed $3 \mathrm{D}$ radial profiles of the density difference. A phase-contrast image contains the 2D distribution of the column density difference integrated along the imaging line, $\tilde{n}_{d}(x, z) \equiv \int n_{d}(\vec{r}) d y$. The excited regions have an

elliptical shape with the same aspect ratio as the trap, $\lambda=f_{z} / f_{r}$, confirming the validity of the local density approximation. Therefore, we can use elliptically averaged profiles of the column density difference, $\tilde{n}_{d}(r)$, to improve the signal-to-noise ratio, where the ellipse for averaging is defined as $x^{2}+\lambda^{2} z^{2}=r^{2}$. The $3 \mathrm{D}$ radial profile $n_{d}(r)$ is calculated using the inverse Abel transformation of $\tilde{n}_{d}(r)$ [22] and $I(r, \Delta \nu) \propto n_{d}(r ; \Delta \nu)$.

With this technique, we obtain homogeneous rf spectra as a function of the 3D position, shown in Fig. 2. These spectra are the main result of this paper and we now discuss their features and implications for our system. The local homogeneous rf spectra shows a spectral gap. The spectral peak is shifted away from the atomic reference line by much more than its line width. Such a gap is not observed in the inhomogeneous rf spectrum (Fig. 1(c)) where the Gaussian wings overlap with the position of the free atomic line. Furthermore, the local rf spectrum reveals an asymmetric line shape of the excitation spectrum. For the central region, the peak is located at $\Delta \nu_{p} \approx 15 \mathrm{kHz}$ and the spectral gap, defined as the minimum energy offset for excitation, is $h \Delta \nu_{g} \approx h \times 10 \mathrm{kHz}$.

The spectral peak position $\Delta \nu_{p}$ in the local rf spectra shows a parabolic dependence on the radial position (Fig.2(a)). This can be explained by unitarity, which demands that all energetic quantities scale with the Fermi energy. At unitarity, the only relevant energy scale in the system is the Fermi energy $\varepsilon_{F} \equiv \hbar^{2}\left(6 \pi^{2} n\right)^{2 / 3} / 2 m[\underline{3}, 23]$, where $n$ is the atom density in one spin state and $m$ is the atomic mass, so the energetic quantities such as chemical potential $\mu$ and pairing gap energy $\Delta$ are proportional to $\varepsilon_{F}$, i.e., $\mu=\xi \varepsilon_{F}$ and $\Delta=\eta \varepsilon_{F}$ with the universal parameters $\xi$ and $\eta$. Therefore the excitation spectrum should also scale with the Fermi energy. In an external harmonic potential $V(r) \propto r^{2}$, the local Fermi energy $\varepsilon_{F}(r)=\mu(r) / \xi=\left(\mu_{0}-V(r)\right) / \xi=\varepsilon_{F 0}\left(1-r^{2} / R^{2}\right)$, where $\mu_{0}$ is the global chemical potential, $\varepsilon_{F 0}$ is the local Fermi energy at the center, $R$ is the radius of the trapped sample and $\varepsilon_{F 0}=\mu_{0} / \xi=V(R) / \xi$. The spectral peak position $\Delta \nu_{p}(r)$ reflects the parabolic radial dependence of the local Fermi energy $\varepsilon_{F}(r)$.

The local Fermi energy at the center is determined from $\varepsilon_{F 0}=\left(R / R_{T F}\right)^{-2} E_{F}$, where $R_{T F}$ is the radial Thomas-Fermi radius for a noninteracting Fermi gas with the same atom number. We obtain $R_{T F}=67.3 \pm 1.1 \mu \mathrm{m}$ for the measured total atom number and trap frequencies. The radius of the trapped sample was measured to be $R=56.6 \pm 1.8 \mu \mathrm{m}$, using absorption images like in Fig. 1(b) and fitting the non-saturated outer wing profile to a zero-temperature TF distribution, giving $\varepsilon_{F 0}=$ $h \times 31.5 \pm 2.5 \mathrm{kHz}$. We estimate the universal parameter $\xi=\left(R / R_{T F}\right)^{4}=0.50 \pm 0.07$, which is in good agreement with previous measurements [4, 5, 24, 25, 26, 27, 28] and Quantum Monte Carlo calculations [29, 30, 31] $(\xi \equiv 1+\beta$ in some references).

The local spectrum at the center of our sample shows 


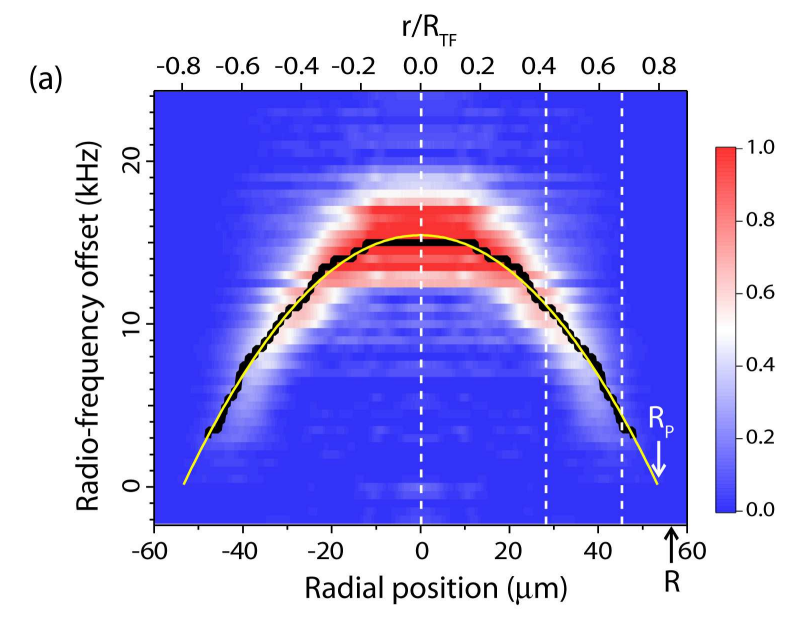

(b)

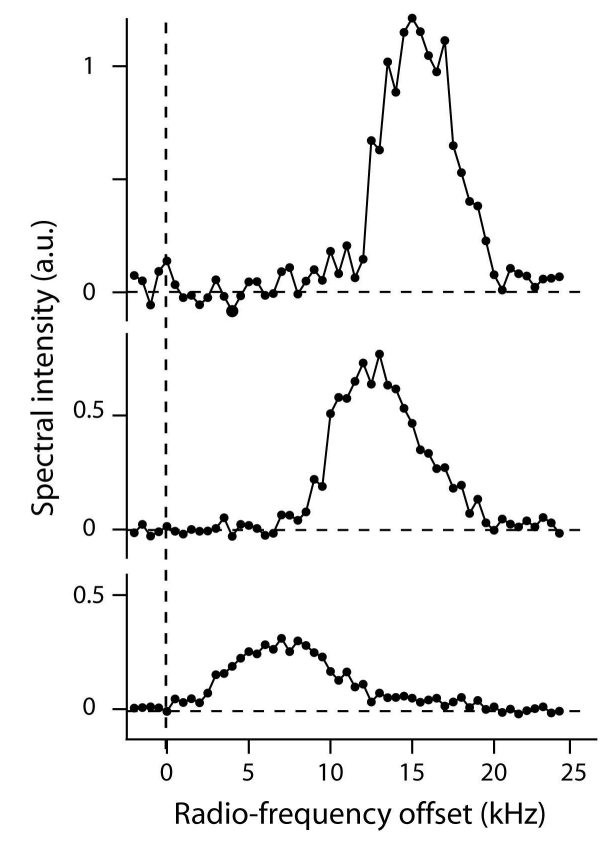

FIG. 2: (color online) Spatially resolved rf spectroscopy of a trapped Fermi gas. (a) The spectral intensity $I(r, \Delta \nu)$ was obtained from the reconstructed 3D profiles of the density difference. See text for the description of the reconstruction method. Local rf spectra are shown for (b) $r=0 \mu m$, (c) $r=24 \mu \mathrm{m}$, and (d) $r=40 \mu \mathrm{m}$, whose positions are marked by vertical dashed lines in (a). Each spectrum is obtained by spatially averaging over $2.5 \mu \mathrm{m}$. $R_{T F}$ is the radial ThomasFermi radius for a noninteracting Fermi gas with the same atom number. The spectral peak position $\Delta \nu_{p}(r)$ in the local rf spectra is marked by the black line in (a). The determination of $\Delta \nu_{p}$ is limited to $r<48 \mu \mathrm{m}$ due to the signal-tonoise ratio. The yellow line is a parabolic fit to $\Delta \nu_{p}(r)$. The radius determined by extrapolating the fit to zero rf offset is $R_{p}=53.6 \mu \mathrm{m}$ indicated by the white down arrow. The black up arrow indicates the radius of the trapped sample, $R=56.6 \mu \mathrm{m}$, measured independently from absorption images like Fig. I(b). the spectral peak at $h \Delta \nu_{p}=0.48(4) \varepsilon_{F 0}$ and the spectral gap of $h \Delta \nu_{g}=0.30(8) \varepsilon_{F 0}$. We determine a radius $R_{p}$ such that $\Delta \nu_{p}\left(R_{p}\right)=0$, extrapolating the parabolic curve fit of $\Delta \nu_{p}$ to zero rf offset (Fig 2(a)). $R_{p}=53.6 \mu \mathrm{m}$ is slightly smaller than the measured radius $R$, which we attribute to finite temperature effects. Previous studies of rf spectroscopy of Fermi gases [10, 11] demonstrated that the spectral peak shifts to higher energy at lower temperature, which is interpreted as the increase of the pairing gap energy. In the outer region of lower density, the local $T / T_{F}$ becomes higher, consequently reducing $h \Delta \nu_{p} / \varepsilon_{F}$. The observation of $R_{p}$ being close to $R$ implies that our experiment is very close to the zero temperature limit. From the relation $T / T_{F}(r) \propto\left(1-r^{2} / R^{2}\right)^{-1}$, we can estimate $T / T_{F}\left(R_{p}\right) \approx 15 \times T / T_{F}(0)$. If we assume that the pairing gap energy starts emerging at $T / T_{F} \approx 0.6\left[14\right.$, we might infer the local $T / T_{F}<0.05$ at the center, close to our measured temperature. Although $h \Delta \nu_{p} / \varepsilon_{F}$ is almost constant over the whole sample, the line width increases in the outer region.

The homogeneous rf spectra measured in our experiment allow a direct comparison with theoretical predictions. However, a comprehensive theoretical interpretation of the rf spectrum of a strongly interacting Fermi gas is not available yet and we discuss here an extrapolation of BCS theory to strong unitarity limited interactions. Rf spectroscopy measures a single-particle spin excitation spectrum, since an rf photon changes the spin state while imparting negligible momentum. The conventional picture of $\mathrm{rf}$ spectroscopy of pairs is a photodissociation process: the initial $|1\rangle-|2\rangle$ bound state, which can be molecules or fermion pairs, breaks into free particles in state $|1\rangle$ and $|3\rangle$. In a BCS superfluid, the free particle in state $|1\rangle$ is regarded as a quasiparticle, so after the spin transition the whole system can be described as the excited BCS state with one quasiparticle and one free particle in state $|3\rangle$. With the assumption of no interactions between state $|1\rangle$ and $|3\rangle$, the rf photon energy offset would be $h \Delta \nu=E_{-k}-\mu+\hbar^{2} k^{2} / 2 m$, where the first term $E_{-k}=\sqrt{\Delta^{2}+\left(\hbar^{2} k^{2} / 2 m-\mu\right)^{2}}$ is the energy cost for generating one quasiparticle excitation with momentum $-k$, the second term is for removing one atom in state $|2\rangle$, and the last term is the kinetic energy of the atom in state $|3\rangle$ with momentum $k$.

The measured FWHM line width is about two times narrower than predicted by the simple model described above. The model spectrum shows a very long tail corresponding to high momentum contributions. This discrepancy might be due to modification of the BCS expressions in the unitarity regime. The narrow peak might imply that the pair wave function is narrower in momentum space and therefore more spatially extended than the BCS prediction. Another extension of our simple model should address the interactions between atoms in state $|1\rangle$ and $|3\rangle$. The mean-field interaction energies due 
to $|1\rangle-|2\rangle$ and $|1\rangle-|3\rangle$ interactions have been empirically assumed to have the same unitarity limited value because of the proximity of a $|1\rangle-|3\rangle$ Feshbach resonance at $\mathrm{B}=690 \mathrm{G}[10,13]$. The recent experiments with imbalanced mixtures and higher densities showed some deviations from this assumption [11].

A localized spin excitation, induced by an rf pulse, eventually diffuses over the sample. This ultimately limits the pulse duration and therefore the spatial resolution. Using phase-contrast imaging, we monitored the evolution of the spatial structure in the excited sample with various delay time after applying an rf pulse. The shell structure was well preserved even after $5 \mathrm{~ms}$ and only some broadening was observed, showing that during the 1-ms pulse the dynamic evolution of the density difference profiles is negligible.

We found that atoms in state $|3\rangle$ rapidly decayed in the sample, although the total atom number difference between state $|1\rangle$ and $|2\rangle$ did not change over time. At the center of the sample where the total atom density is about $8 \times 10^{12} \mathrm{~cm}^{-3}$, the life time of atoms in state $|3\rangle$ was measured to be less than $0.4 \mathrm{~ms}$. Since a $|1\rangle-|3\rangle$ mixture and a $|2\rangle-|3\rangle$ mixture are stable at $B=833 \mathrm{G}$ [32], the decay should be associated with exoergic molecule formation via three-body collisions involving one atom from each spin state. We observed that the loss of one atom in state $|3\rangle$ was accompanied by loss of one atom in state $|1\rangle$ and one atom in state $|2\rangle$, supporting the three-body loss mechanism 33.

Our new technique of spatially resolved rf spectroscopy should be able to address the important questions also at finite temperature. One question is whether the observed double peak structure [10, 11] of an atomic line and a pairing peak is purely inhomogeneous, or whether it is possible to have local coexistence of paired and unpaired atoms. This is of course possible on the BEC side of the Feshbach resonance where in a certain temperature range, bound molecules and thermally dissociated free atoms coexist, but it is an open question, how this picture will change in the BEC-BCS crossover.

In conclusion, we present spatially resolved rf spectroscopy of a trapped Fermi gas, using the in situ phasecontrast imaging technique. The homogeneous rf spectra of a Fermi gas at unitarity provide a benchmark for a complete theoretical description, which should reveal microscopic details of the paired states.

We thank M. Zwierlein and R. Grimm for stimulating discussions and T. Pasquini for critical reading of the manuscript. This work was supported by the NSF and ONR.

\footnotetext{
* Electronic address: yishin@mit.edu
}

[1] M. W. Zwierlein et al., Nature 435, 1047 (2005).

[2] R. Grimm, cond-mat/0703091 (Proceedings of the International School of Physics "Enrico Fermi", Course CLXIV) (2007).

[3] T.-L. Ho, Phys. Rev. Lett. 92, 090402 (2004).

[4] J. Kinast et al., Science 307, 1296 (2005).

[5] J. T. Stewart, J. P. Gaebler, C. A. Regal, and D. S. Jin, Phys. Rev. Lett. 97, 220406 (2006).

[6] A. Altmeyer et al., Phys. Rev. Lett. 98, 040401 (2007).

[7] C. A. Regal and D. S. Jin, Phys. Rev. Lett. 90, 230404 (2003).

[8] S. Gupta et al., Science 300, 1723 (2003).

[9] C. A. Regal et al., Nature 424, 47 (2003).

[10] C. Chin et al., Science 305, 1128 (2004).

[11] C. Schunck et al., Science 316, 867 (2006).

[12] M. Greiner, C. A. Regal, and D. S. Jin, Phys. Rev. Lett. 94, 070403 (2005).

[13] J. Kinnunen, M. Rodríguez, and P. Törmä, Science 305, 1131 (2004).

[14] Y. He, Q. Chen, and K. Levin, Phys. Rev. A 72, 011602(R) (2005).

[15] Y. Ohashi and A. Griffin, Phys. Rev. A 72, 063606 (2005).

[16] Z. Yu and G. Baym, Phys. Rev. A 73, 063601 (2006).

[17] Y. Shin, M. W. Zwierlein, C. H. Schunck, A. Schirotzek, and W. Ketterle, Phys. Rev. Lett. 97, 030401 (2006).

[18] Z. Hadzibabic et al., Phys. Rev. Lett. 91, 160401 (2003).

[19] Note that also atoms in state $|3\rangle$ contribute to the optical signal. However, since their density is proportional to $n_{d}$, the signal is still proportional to $n_{d}$.

[20] The measured $h \Delta \nu_{i} / E_{F} \approx 0.45$ is higher than the values reported in Ref. [10, 11], which we attribute to lower temperature and less heating over the short pulse duration.

[21] The magnetic field was calibrated by determining the resonance frequency $\nu_{0}$ of the $|2\rangle-|3\rangle$ transition in the absence of atoms in state $|1\rangle \cdot \nu_{0}=81.700(1) \mathrm{MHz}$ corresponds to a magnetic field of about 833.0(2) G.

[22] R. N. Bracewell, The Fourier Transform and Its Applications (McGraw-Hill, 1986).

[23] H. Heiselberg, Phys. Rev. A 63, 043606 (2001).

[24] K. M. O'Hara et al., Science 298, 2179 (2002).

[25] M. E. Gehm, S. L. Hemmer, S. R. Granade, K. M. O'Hara, and J. E. Thomas, Phys. Rev. A 68, 011401(R) (2003).

[26] M. Bartenstein et al., Phys. Rev. Lett. 92, 120401 (2004).

[27] T. Bourdel et al., Phys. Rev. Lett. 93, 050401 (2004).

[28] G. B. Partridge et al., Science 311, 503 (2006).

[29] J. Carlson, S.-Y. Chang, V. R. Pandharipande, and K. E. Schmidt, Phys. Rev. Lett. 91, 050401 (2003).

[30] G. E. Astrakharchik, J. Boronat, J. Casulleras, and S. Giorgini, Phys. Rev. Lett. 93, 200404 (2004).

[31] J. Carlson and S. Reddy, Phys. Rev. Lett. 95, 060401 (2005).

[32] R. Grimm (private communication).

[33] In our experiment, the maximum fractional atom loss in state $|2\rangle(|1\rangle)$ was about $30 \%(15 \%)$ at $\Delta \nu \approx 10 \mathrm{kHz}$, which could not be reduced further due to the signal-tonoise ratio. Including the spatial width of the excitation region, we estimate that the local density depletion was about $45 \%$. 